\title{
Large Scale Outflow from a Radio Loud AGN in Merging Galaxies at Redshift 2.48
}

\author{
Hsin-Yi Shih ${ }^{1}$ and Alan Stockton ${ }^{2}$ \\ ${ }^{1}$ Gemini Observatory, \\ 670 N. Aohoku Pl, Hilo, HI, 96720, United States \\ email: jshih@gemini.edu \\ ${ }^{2}$ Institute for Astronomy, University of Hawaii, \\ 2680 Woodlawn Dr. Honolulu, HI, 96822, United States \\ email: stockton@ifa.hawaii.edu
}

\begin{abstract}
We present observations of a remarkable compact group of galaxies at $z=2.48$. Four galaxies, all within $40 \mathrm{kpc}$ of each other, surround a powerful high redshift radio source. This group comprises two compact red passive galaxies and a pair of merging galaxies. One of the red galaxies, with an apparent stellar mass of $3.6 \times 10^{11} M_{\odot}$ and an effective radius of $470 \mathrm{pc}$, is one of the most extreme examples of a massive quiescent compact galaxy found so far. One of the pair of merging galaxies hosts the AGN producing the large powerful radio structure. The merger is massive and enriched, consistent with the mass-metallicity relation expected at this redshift. Close to the merging nuclei, the emission lines exhibit broad and asymmetric profiles that suggest outflows powered either by a very young expanding radio jet or by AGN radiation. At $\gtrsim 50 \mathrm{kpc}$ from the system, we found a fainter extended-emission region that may be a part of a radio jet-driven outflow.
\end{abstract}

Keywords. galaxies: evolution, galaxies: high-redshift, galaxies: interactions, galaxies: jets,galaxies: active

\section{Introduction}

While carrying out a search for massive, passively evolving galaxies in high-redshift radio-source fields, we have come across a compact group of massive galaxies in the field of the radio source TXS2332+154, at $z=2.48$ (De Breuck et al. 2001). Given that isolated compact groups of galaxies at such a high redshift are likely quite rare, we followup on the TXS2332+154 group with a series of imaging and spectroscopy.

\section{Imaging}

With ground-based telescopes, we have obtained $J, H$, and $K^{\prime}$ imaging with the CISCO IR camera on the Subaru $8.2 \mathrm{~m}$ telescope, $i^{\prime}$ imaging with the Gemini Multi-Object Spectrograph (GMOS) on the Gemini North telescope, and I imaging with the LowResolution Imaging Spectrograph (LRIS) on the Keck I telescope. We also obtained deep imaging of the TXS2332+154 field with the Spitzer Space Telescope, including all 4 Infrared Array Camera (IRAC) bands. Photometry derived from these images are used to constrain the SEDs of each galaxy in the group.

In addition to these observations obtained to define the spectral-energy distribution (SED), we also obtained laser-guide-star adaptive-optics (LGSAO) $K^{\prime}$ imaging with the NIRC2 camera on the Keck II telescope to obtain high-resolution morphological information. The image is shown in Figure 1, with the galaxies labeled G1 - G4. 


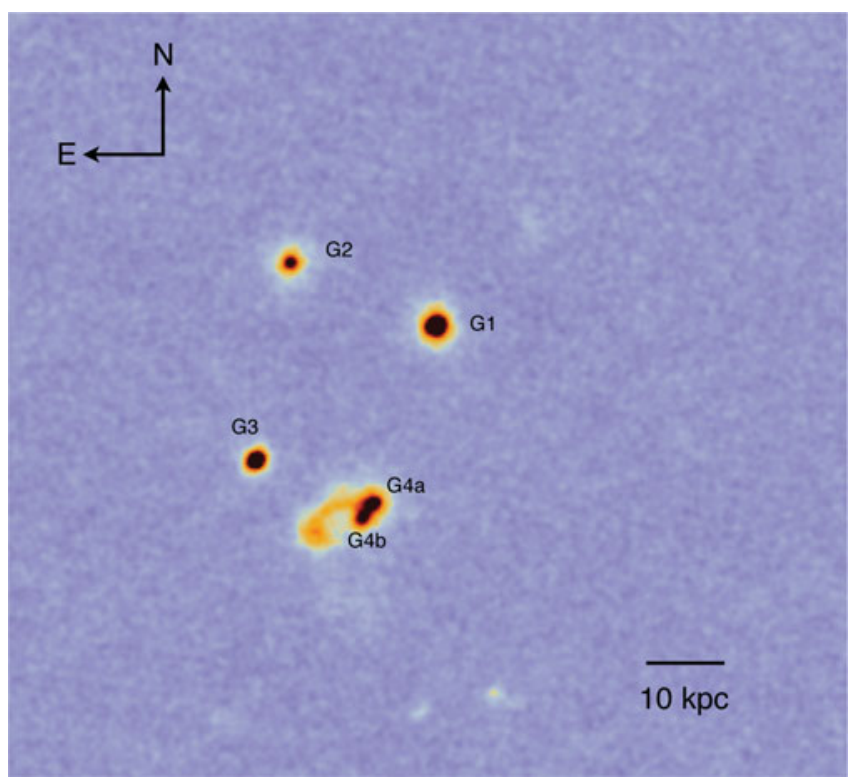

Figure 1. Keck II LGSAO image of the compact group.

Figure 2 shows all the photometries obtained superposed on best-fitting Bruzual \& Charlot (2003) models. Fitting the profile of each galaxy in the LGSAO image and the SED gives the estimated size, mass, and stellar population age for each galaxy. In summary, G2 and G3 both have stellar population ages of $\sim 2$ Gyr old, and stellar mass of $3.6 \times 10^{11} M_{\odot}$. G4 clearly comprises two merging galaxies which have a combined mass of $2.5 \times 10^{11} M_{\odot}$, and a much younger stellar population age of $72-720 \mathrm{Myr}$, indicating a recent starburst. The large uncertainty in G4's SED comes from the difficulty in subtracting the contribution from strong emission lines. No adequate SED fit for G1 could be found for the radio-source redshift of $z=2.48$, hinting that it is likely at a different redshift.

Out of all the galaxies, G3 has the smallest effective radius of $R_{\mathrm{e}}$ of only $470 \mathrm{pc}$, indicating an extremely high stellar density. Though G2 has similar morphology and stellar mass as G3, its $R_{\mathrm{e}}$, at $1.83 \mathrm{kpc}$, is much larger than that of G3. Although many elliptical galaxies at $z \sim 2$ are much more compact than their present day counterparts (e.g., Van Dokkum et al. 2008), G3 represent one of the most extreme example of compact red galaxies studied so far.

\section{Spectroscopy}

We have long-slit NIR (rest-frame optical) and optical (rest-frame UV) spectra of the compact group members at the slit positions shown in Figure $3 a$. We obtained $H$-band spectra of two of the compact objects in the field (G1 and G3) using MOSFIRE on Keck. We also have a Gemini GNIRS cross-dispersed spectrum of the interacting pair (G4). In the optical, we have three sets of spectroscopy from GMOS on Gemini North, FOCAS on Subaru, and LRIS on Keck I. The spectral coverage of each galaxy and the most important features in the above spectra are shown in Figure $3 a$.

In brief, the spectroscopy has confirmed that galaxy G3 and the interacting pair G4 are at $z=2.482 \pm 0.001$ and $z=2.469 \pm 0.002$ respectively, but that galaxy $\mathrm{G} 1$ is a foreground interloper at $z=1.061 \pm 0.002$. 


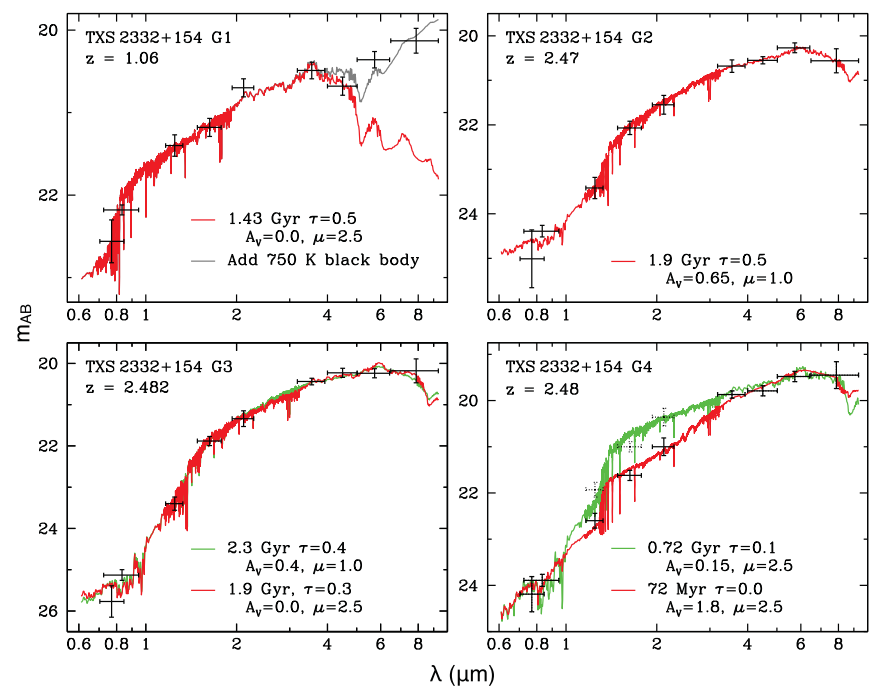

Figure 2. SED photometry and models for the galaxies in the TXS2332+154 group. From left to right, the photometric bands are $i^{\prime}, I_{c}, J, H, K^{\prime}$, IRAC $3.6 \mu \mathrm{m}, 4.5 \mu \mathrm{m}, 5.7 \mu \mathrm{m}$, and $7.9 \mu \mathrm{m}$. The models shown are the best-fit exponentially decreasing star-formation models (with time constant $\tau$ given in Gyr) among those with metallicities $\mu=0.4,1.0$, or 2.5 solar and Calzetti et al. (2000) reddening corresponding to extinctions $A_{V}$ ranging from 0 to 2.0 .

\subsection{Extended Lya Emission and Nuclear Outflow}

The LRIS spectrum of interacting pair (G4) shows bright extended Ly $\alpha$ emission (Figure $3 b$ ), which spans a $\sim 20 \mathrm{kpc}$ region along the slit, almost extending out to the position of G1. In the GNIRS spectrum, the [OIII] emission from the nuclear regions shows a clear asymmetry, which requires two Gaussian components to fit. The dominant component of the best fit model has $F W H M=255 \pm 15 \mathrm{~km} \mathrm{~s}^{-1}$, and is accompanied by a fainter component that is slightly broader, with $F W H M=320 \pm 70 \mathrm{~km} \mathrm{~s}^{-1}$ and blueshifted by $-290 \pm 45 \mathrm{~km} \mathrm{~s}^{-1}$. This blueshifted component is likely part of an AGN driven outflow.

The brightest radio component of TXS2332+154 is compact and has a steep spectral index. Therefore, it can be considered a compact-steep-spectrum (CSS) source, which is usually associated with young radio sources (ODea 1998). CSS sources similar to TXS2332+154, but at at lower redshifts, are known to drive outflows with complex velocity structures, often involving a broad and centrally concentrated component (e.g., Shih et al. 2013). Therefore, young emerging radio jet(s) impacting the surrounding is the most likely dominant driving mechanism for the nuclear outflow. The same outflow may also be responsible for the extended Ly $\alpha$ emission northwest of the galaxies.

\subsection{Extended Emission-Line Region}

In addition to the bright extended emission lines close to the merger nuclei, we have detected fainter extended [OIII] $\lambda 5007$ emission on the north-west side of G1 in the MOSFIRE spectrum (Figure $3 e$ ). As we have eliminated G1 as a member of the $z=2.48$ group, a hidden quasar in the merging pair G4-located $>50 \mathrm{kpc}$ from the tip of the detected [OIII] emission-becomes the only likely ionizing source. The gas is more redshifted in the southeast and more blue-shifted in the northwest, spanning a velocity range of -140 to $-240 \pm 20 \mathrm{~km} \mathrm{~s}^{-1}$ with respect to the dominant component of the nuclear emission. The gas velocity dispersion is relatively low, with $F W H M=35 \pm 15 \mathrm{~km} \mathrm{~s}^{-1}$ over the detected range. TXS2332+154 has an extended radio jet component that has 

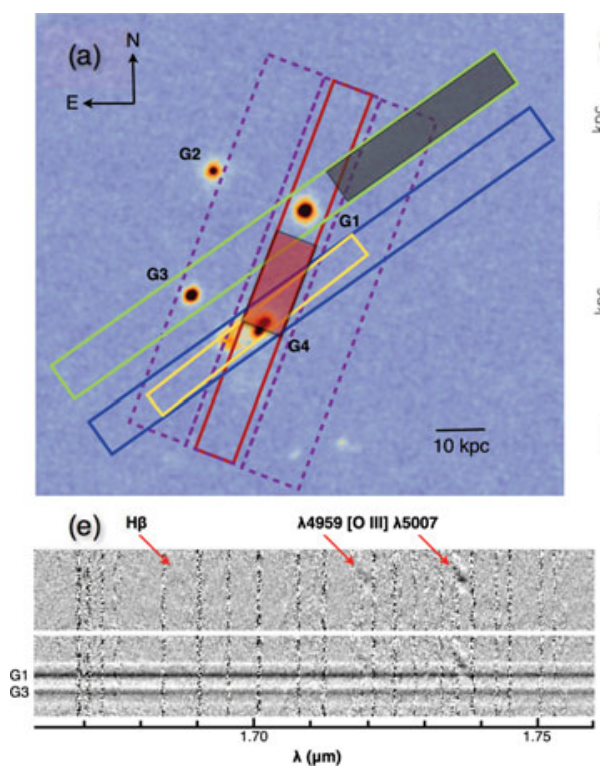
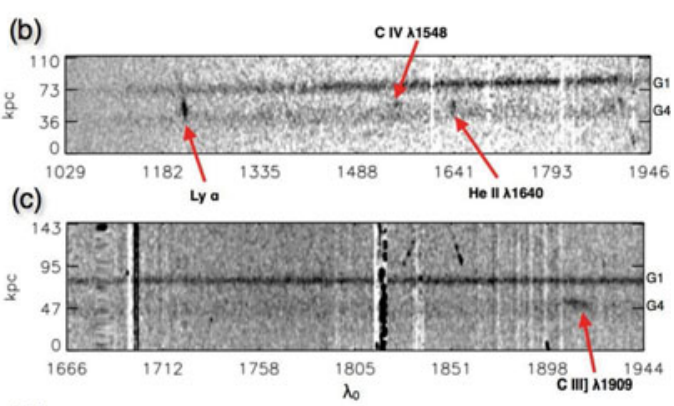

(d)

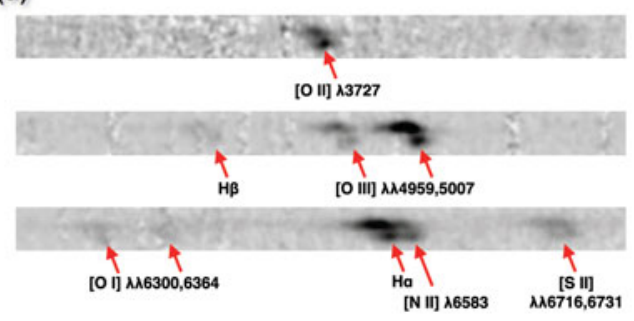

Figure 3. Spectroscopy of the TXS2332+154 field. (a) The position and slit width of the NIR and optical spectra overlaid on the NIRC2 LGSAO image. The MOSFIRE slit is shown in green with the gray shaded region indicating where [OIII] extended emission is detected. The GNIRS slit is shown in yellow. The slits for the optical spectra are shown as follows: purple for GMOS (including the offset positions), red for LRIS, and blue for the FOCAS. The red shaded region indicate where extended Ly $\alpha$ emission is detected with LRIS. (b) The 2-D LRIS spectrum showing the strong extended Ly $\alpha$ feature. (c) The 2-D GMOS spectrum showing the broad [CIII] emission. (d) Parts of the GNIRS echellete spectrum showing regions around [OII] $\lambda 3727$, [OIII] $\lambda \lambda 4959,5007+\mathrm{H} \beta$, and $\mathrm{H} \alpha+[\mathrm{NII}] \lambda 6548,6583$. The upper trace in each spectrum is the central part of the G4 pair; the lower trace shows emission in the tidal tail. (e) The 2-D MOSFIRE spectrum at the green slit position shown in $a$, showing only the $\mathrm{H} \beta$ - [OIII] region at $z \sim 2.47$. The upper plot has had a model of the positive and negative continuum features produced by the MOSFIRE pipeline subtracted in order to show the emission more clearly.

a similar physical scale as the extended ionized gas. Therefore, the extended gas cloud may part of a jet-driven outflow.

\section{References}

Bruzual, G., \& Charlot, S. 2003, MNRAS, 344, 1000

De Breuck, C., van Breugel, W., Rttgering, H., Stern, D., Miley, G., de Vries, W., Stanford, S. A., Kurk, J., \& Overzier, R. 2001, AJ, 121, 1241

Calzetti, D., Armus, L., Bohlin, R. C., Kinney, A. L., Koornneef, J., \& Storchi-Bergmann, T. 2000, ApJ, 533, 682

ODea, C. P. 1998, PASP, 110, 493

Shih, H.-Y., Stockton, A., \& Kewley, L. 2013, ApJ, 772, 138

van Dokkum, P. G., Franx, M., Kriek, M., Holden, B., Illingworth, G. D., Magee, D., Bouwens, R., Marchesini, D., Quadri, R., Rudnick, G.,Taylor, E. N., \& Toft, S. 2008, ApJ, 677, L5 